\title{
The Design of Low Pass Filter with Active Filter based on Complex Surroundings
}

\author{
Changhai $\mathrm{Li}^{1, \text { a }} \quad \mathrm{Su} \mathrm{Ye} \mathrm{C}^{2}$ Zhihui Sun $^{1} \quad$ Yuantao $\mathrm{Yu}^{1}$ \\ 1Aviation Lifesaving Dep, Air Force Aviation University, \\ 2Air Force Aviation University, Changchun, Jilin, 130022, China \\ Ich19731008@163.com,
}

Keywords: Disturb; Active Filter; Low Pass Filter;

\begin{abstract}
Because of the effects of the wind-noise, sound-noise, strong electromagnetic interfere, sensor itself, and the magnified circuit etc. at the survey spot, the received transducer signals often include noise signals of multi-frequency disturbance. Under serious circumstances, such noise signals would submerge the input signal need-extracted, therefore resulting the system cannot obtain the surveyed signals. Filter measures should be adopted, noise should be exterminated by signal filter, so that to enhance SNR of the system. When high-speed dropping parachutist issue orders, when recording at noise meeting spot, or other such complex surroundings, filter of such functions is needed, it can also be applied in area of message technology treatment of military and civil use.
\end{abstract}

\section{Introduction}

The active components in active filter can adopt crystal transistors, and operational amplifier. The active filter composed of operational amplifier has the merits of small volume, light weight, low loss. And it also provides some gain, and the cushioning effect, so it is widespread used.

\section{Design of the Chip Kick Mechanics}

1.The frequency feature of low pass filter

The useful signals of this system are at low frequency bound, but the disturb signals are at high frequency bound. The Low Pass Filter (LPF) is a kind of which, through low frequency signals, can restrain or attenuate high frequency signals. The frequency feature of LPF is as shown in figure 1.

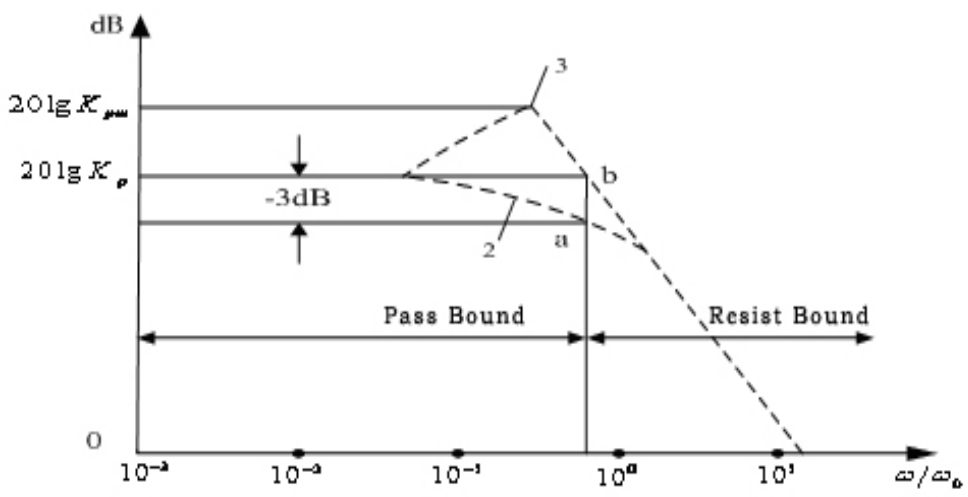

figure 1: The frequency feature of low pass filter

In figure 1, solid line indicates ideal frequency respond, yet dotted line indicates the actual feature curve. In this figure, the frequency bound which allowing signals pass through is $0-\omega_{0}$, this frequency bound is called Pass Bound of LPF, the bound which don't allow signals pass though $\left(\omega>\omega_{0}\right)$ is called Resist Bound. $\omega_{0}=2 \pi f_{0}$ is called End-angle frequency, fo is called End frequency. Curve 2 in figure has no Resonance Peak in the pass bound, when the gain drops to 
$K_{p} / \sqrt{2}$ (i.e. gain is reduced $3 \mathrm{db}$ ), then the frequency is End-frequency, corresponding to a point. Curve 3 in figure has Resonance Peak in the pass bound, at this moment, it is stipulated, the scope feature from peak value $K_{p m}$ comes back to initial value $K_{p}$, the frequency is End frequency, corresponding to $\mathrm{b}$ point.

2.The design of the Active Filter

This experiment device adopts two-shift low pass active filter. The circuit is shown as figure 2 .

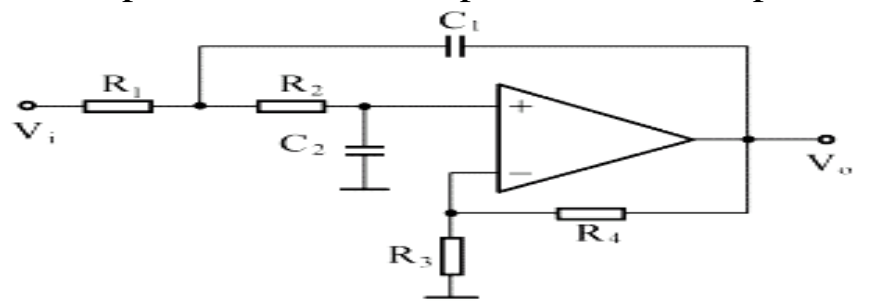

figure 2 :two-shift low pass active filter.

(1) basic feature:

The transfer function is

$$
K(s)=\frac{\frac{1}{R_{1} R_{2} C_{1} C_{2}} \times \frac{R_{1}+R_{2}}{R_{3}}}{s^{2}+s\left[\frac{1}{R_{1} C_{1}}+\frac{1}{R_{2} C_{2}}+\frac{1-A_{f}}{R_{2} C_{2}}\right]+\frac{1}{R_{1} R_{2} C_{1} C_{2}}}
$$

The contrast with low pass transfer function according to two-shift standard has

$$
\left\{\begin{array}{l}
K_{P}=1+\frac{R_{f}}{R_{3}} \\
\omega_{0}=\sqrt{\frac{1}{R_{1} R_{2} C_{1} C_{2}}} \\
\xi=\frac{1}{2}\left[\sqrt{\frac{R_{2} C_{2}}{R_{1} C_{1}}}+\sqrt{\frac{R_{1} C_{2}}{R_{2} C_{1}}}+\left(1-K_{P}\right) \sqrt{\frac{R_{1} C_{1}}{R_{2} C_{2}}}\right]
\end{array}\right.
$$

If $\xi<1 / \sqrt{2}$, then Resonance Peak appears in the scope feature. In order to get the angle frequency at Resonance Peak, the following formula

$$
K(\omega)=\frac{K_{P}}{\sqrt{\left(1-\frac{\omega^{2}}{\omega_{0}^{2}}\right)^{2}+\left(2 \xi \frac{\omega}{\omega_{0}^{2}}\right)^{2}}}
$$

To $\omega$ differential and set it equal to 0 , then get

$$
\frac{d}{d \omega}\left[\left(1-\frac{\omega^{2}}{\omega_{0}^{2}}\right)^{2}+\left(2 \xi \frac{\omega}{\omega_{0}^{2}}\right)^{2}\right]=0
$$

Arrange it

$$
4 \frac{\omega}{\omega_{0}^{2}}\left(\frac{\omega^{2}}{\omega_{0}^{2}}+2 \xi^{2}-1\right)_{=0}
$$

From this we can get the angle frequency at Resonance Peak 


$$
\omega_{P} \omega=\omega_{P}=\omega_{0} \sqrt{1-2 \xi^{2}}
$$

(4)

The maximum Peak Value corresponding to ${ }^{\omega}{ }_{P}$ is

$$
K_{p m}=K\left(\omega_{p}\right)=\frac{K_{p}}{2 \xi \sqrt{1-\xi^{2}}}
$$

When

$$
\xi=1 / \sqrt{2} \approx 0.7
$$

Formula

$$
K(\omega)=\frac{K_{p}}{\sqrt{\left(1-\frac{\omega^{2}}{\omega_{0}^{2}}\right)^{2}+\left(2 \xi \frac{\omega_{0}}{\omega}\right)^{2}}}
$$

Turn to

$$
K(\omega)=\frac{K_{p}}{\sqrt{1+\left(\frac{\omega}{\omega_{0}}\right)^{4}}}
$$

Then no Peak Value appears in the scope feature.

When $\omega=\omega_{0}$ : $K(\omega)=K_{p} / \sqrt{2}$. It is clear when $\omega=\omega_{0}$ the scope feature descends 3dB; in other word, when $\xi=1 / \sqrt{2} \approx 0.7$ end angle frequency $\omega_{c} \quad$ is equal to inherent angle frequency $\omega_{0}$, that is $\omega_{c}=\omega_{0}$ 。

When $\xi<1 / \sqrt{2}$, the scope feature has peak value, then end angle frequency is defined as the scope feature from Peak Value back to original value angle frequency. Therefore only let the denominator in formula (2) equal to 1 , then we can get end angle frequency

$$
\omega_{c}=\omega_{0} \sqrt{1-2 \xi^{2}}
$$

The difference between peak value and original value

$$
20 \lg K_{p m}-20 \lg K_{p}=20 \lg \frac{K_{p m}}{K_{p}}=20 \lg \frac{1}{2 \xi \sqrt{1-\xi^{2}}}
$$

When $\xi<1 / \sqrt{2}$, two-shift active filter $\xi$ and $20 \lg \frac{K_{p m}}{K_{p}} \quad(\mathrm{~dB})$, the parameter relation is as in figure 3 .

\begin{tabular}{|c|l|l|}
\hline$\xi$ & $20 \lg \frac{K_{p m}}{K_{p}}(\mathrm{~dB})$ & $\omega_{\mathrm{c}}$ \\
\cline { 2 - 3 } & 0.500 & two-shift low pass \\
\hline 0.5792 & 0.512 & $0.8114{ }^{\omega_{0}}$ \\
\hline 0.5774 & 1.000 & $0.8165{ }^{\omega_{0}}$ \\
\hline 0.5227 & 1.248 & $1.00000^{\omega_{0}}$ \\
\hline 0.5000 &
\end{tabular}




\begin{tabular}{|l|l|l|}
\hline 0.4434 & 2.000 & $1.10200^{\omega_{0}}$ \\
\hline 0.3882 & 3.000 & $1.18800^{\omega_{0}}$ \\
\hline
\end{tabular}

Form 3: when $\xi<1 / \sqrt{2}$ two-shift filter parameter

(2 ) Design Steps

When making a concrete design of Active Filter, according to the feature require of concrete filter, choose proper inherent frequency ${ }^{\omega}{ }_{0}$ and damping coefficient $\xi$ and pass bound $K_{p}$, and then calculate the value of passive elements in the circuit. Considering that the known conditions are less than the unknown, we often pre-choose condenser $C_{1}$ and electric capacity proportion coefficient $m=C_{1} / C_{2}$, thus we can calculate the passive elements value according to following steps.

The first step, pre-choose value of condenser $C_{1}$.

\begin{tabular}{|c|c|c|c|}
\hline$f_{0} / \mathrm{Hz}$ & $C_{1} / \mu \mathrm{F}$ & $f_{0} / \mathrm{Hz}$ & $C_{1} / \mu \mathrm{F}$ \\
\hline $10^{0} \sim 10^{1}$ & $\begin{array}{c}2 \times 10^{1} \sim \\
10^{0}\end{array}$ & $10^{3} \sim 10^{4}$ & $10^{4} \sim 10^{3}$ \\
\hline $10^{0} \sim 10^{1}$ & $10^{0} \sim 10^{-1}$ & $10^{4} \sim 10^{5}$ & $10^{3} \sim 10^{2}$ \\
\hline $10^{0} \sim 10^{1}$ & $10^{-1} \sim 10^{-2}$ & $10^{5} \sim 10^{6}$ & $10^{2} \sim 10^{1}$ \\
\hline
\end{tabular}

Form 4. $f_{0}$ and $C_{1}$ corresponding relation form

The second step, according to the chosen condenser $C_{1}$ and proportion coefficient $\mathrm{m}$, calculate condenser $C_{2}$ value $C_{2}=m C_{1}$

The third step, from Form (7) we can get

$$
R_{1}=\frac{1}{m C_{1}^{2} \omega_{0}^{2} R_{2}}
$$

Make form (9 ) into (7), further can calculate

$$
R_{2}=\frac{\xi}{m C_{1} \omega_{0}}\left[1+\sqrt{\frac{K_{p}-m-1}{\xi^{2}}}\right]
$$

Evidently electric capacity proportion coefficientm must set content with

$$
\begin{aligned}
& \frac{K_{p}-m-1}{\xi^{2}} \geq-1 \\
& \text { i.e. } \quad m \leq K_{p}-1+\xi_{2}
\end{aligned}
$$

The forth step, according to the known $K_{p}$ all values and reducing transport, set, input bias electric circuit and the require of reducing drift, determine $R_{i}$ $R_{f}$ 。 
from $K_{p=1+} \frac{R_{f}}{R_{3}}$

And $R_{1} R_{2}=R_{f} / R_{3}$

$$
\text { i.e. }\left\{\begin{array}{l}
R_{f}=K_{p}\left(R_{1}+R_{2}\right) \\
R_{3}=\frac{R_{f}}{K_{p}-1}
\end{array}\right.
$$

(3) the filter designed in this system

In this system, the designed two-shift Active Low Pass Filter $K_{p=10}, f_{0}=1000 \mathrm{~Hz}$, $\xi<1 / \sqrt{2}$, The process of calculating the value of passive elements in the two-shift active low pass filter is as follows: because of $\xi<1 / \sqrt{2}$, i.e. there is no resonance peak in the scope feature, the end frequency is equal to inherent frequency of the filter. Then $f_{c}=f_{0}=1000 \mathrm{~Hz}$ 。

According to $f_{0}$, from form 2-2, choose $C_{1}=0.01 \mu \mathrm{F}$, and make $\mathrm{m}=2, \quad$ then $C_{2}=m C_{1}=0.02$ $\mu \mathrm{F}$

$$
\begin{aligned}
& R_{2}=\frac{\xi}{m C_{1} \omega_{0}}\left[1+\sqrt{\frac{K_{p}-m-1}{\xi^{2}}}\right]=\frac{1}{2 \sqrt{2} \times 0.01 \times 10^{-6} \times 2 \pi \times 1000}\left[1+\sqrt{1+\frac{10-1-2}{(1 / \sqrt{2})^{2}}}\right] \approx 27.4(K \Omega) \\
& R_{1}=\frac{1}{m C_{1}^{2} \omega_{0}^{2} R_{2}} \\
& =\frac{1}{2 \times\left(10^{-8}\right)^{2} \times 4 \pi^{2} \times 10^{6} \times 27.4 \times 10^{3}} \approx 320(K \Omega) \\
& R_{f}=K_{p}\left(R_{1}+R_{2}\right)=10(4.62+27.4) \approx 320(K \Omega) \quad R_{3}=\frac{R_{f}}{K_{p}-1}=\frac{320}{10-1} \approx 35.6(K \Omega)
\end{aligned}
$$

\section{References}

[1] Welter, GG, A samPling theoren, IEEE Trans. on Information Theory , VOI.38, 1992,pp,881-883

[2] Advantech Corp. Total Solution for PC-based Industrial Automation. 1996

[3] Advantech Corp. PC-LabCard User annual. 1995,10

[4] HummelR, Moniot R. Reconstruction from zero-crossing in scale space[J].IEEE Trans. Acoust. Speech Signal Processing, vol.3,No.12, pp.2111-2130,1989

[5] Lu J. Healy DM, Wcaver JB. Signal recovery and wavelet reproducing kemels[J]. IEEE Trans. Signal Processing, vol.42 , No.7 , pp . 1845-1848 , 1994

[6]Cetin AE, Ansari R. Signal recovery from wavelet transform maxima[J]. IEEE Trans. on Signal procesing, vol.42, No.l 1994

[7] Welter, GG, A samPling theoren, IEEE Trans. on Information Theory , VOI.38, 1992,pp,881-883 